\title{
NOVEL ADAPTIVE FIXTURING FOR PRECISE MICROPOSITIONING OF THIN WALLED PARTS
}

\author{
A. Merlo* \\ $R \& D$, \\ Advanced Engineering \\ and Materials Department \\ Ce.S.I., Centro Studi Industriali \\ Cologno Monzese (MI), \\ 10 Via Tintoretto, 20093 \\ Italy \\ Email: merlo@cesi.net
}

\author{
D. Ricciardi \\ E. Salvi \\ S. De Cristofaro \\ $R \& D$, \\ Advanced Engineering \\ and Materials Department \\ Ce.S.I., Centro Studi Industriali \\ Cologno Monzese (MI), \\ 10 Via Tintoretto, 20093 \\ Italy
}

\author{
F. Aggogeri \\ N. Pellegrini
}

Department of Mechanical

and Industrial Engineering

University of Brescia

Brescia (BS),

38 Via Branze, 25123

Italy

\section{ABSTRACT}

Fixtures are used to locate and hold workpieces during machining. Because workpiece surface errors and fixture set-up errors (called source errors) always exist, the fixtured workpiece will consequently have position and/or orientation errors (called resultant errors) that will definitely affect the final machining accuracy.

This paper illustrates a novel adaptive fixturing based on active clamping forces for smart micropositioning of thin walled precision parts. The aim of obtaining a modular unit, reusable and exploitable to different industrial applications has been pursued during the design phase. The proposed adaptive fixturing device can lead to the following advantages:

- to perform an automatic errors-free workpiece clamping and then drastically reduce the overall fixturing set up time;

- to recover unwanted strains induced on the workpiece, in order to limit the amplitude of elastic strain recovery;

- to perform, if necessary, active vibration control (AVC) in order to limit vibration/chatter effects induced by the cutting tool.

\footnotetext{
*Address all correspondence to this author.
}

\section{INTRODUCTION AND STATE OF THE ART}

There is a growing demand for product miniaturisation that requires the development of direct and indirect methods for manufacturing parts and tooling inserts that incorporate thin features.

One of the viable manufacturing routes for producing metal components that incorporate such thin features is their machining from a solid workpiece. Especially, it is a promising route for manufacturing complex microengineering structures and thus reduces significantly the complexity of followup assembly/integration operations. While at "macro" scale this could be achieved by assembling the products from thin section components there is not a viable alternative approach for their manufacture at "micro" scale. Micromilling of metal structures with "thin" features represent a major challenge towards broadening the use of this technology in a range of microengineering applications, for example in producing multi-channels micro structures, housing for mechanical microdevices and surgical instruments. The main factor preventing the broader use of micromilling for producing microstructures incorporating thin features from a solid workpiece is the stability of the machining operation. By making ribs and webs thinner, their stiffness decreases which could result in the occurrence of vibrations during machining. As a consequence the process accuracy deteriorates 
and could lead to manufacture of substandard components. The high frequency vibrations that could occur during micromilling result in poor surface quality. Corrections through follow-up finishing operations are not possible or cost effective due to the feature sizes. Traditional fixtures which have been used for many years are not able to meet the requirements of modern manufacturing processes.

The design of a novel adaptive fixturing system implies the merging of many disciplines such as Mechanics, Electronics and Control, resulting in the definition of Mechatronic. This paper deals with the mechatronic design of an adaptive fixture able to engage and fix different workpieces to drastically save positioning time, and to provide vibration suppression in closed control loop during the machining phase. Several mechatronic systems were designed with similar aims . In [1] and [2] an adaptronic chuck for precision positioning in lathes together with an optical measurement system and best fit algorithms is presented. This solution offers the ability to compensate eccentricities by active clamping adjustment. Integrated piezoactuators are controlled by microcontrollers which communicate with an external control desk via Bluetooth. A positioning range of $\pm 100 \mu \mathrm{m}$ in two degrees of freedom can be achieved. In order to control pressure in the clamping cylinders of chucks, force sensors were also integrated [3]. Thus, the centripetal forces could be compensated to make a sensitive clamping possible. These technologies focus on rotational symmetric parts, and not on free form part. A smart fixture system based on magneto-rheologic fluids has been developed in [4]. The fluid is in direct contact with the held part. A magnetic field increases the viscosity of the fluid allowing the application of clamping forces. In [5] a fixturing device with dynamic clamping forces is proposed. The forces are generated by linear actuator coupled with a servo motor. Both fixturing devices presented in [4] and [5] are quite big (low power density), and do not provide the automatic recovery of errors/distortions which arise during clamping obtainable with the new proposed one.

Beyond this, the proposed innovative solution refers to an intelligent fixture for complex free formed and thin walled meso/micro parts. This device can recover in real-time, by means of the closed loop control, the micropositioning errors and distortions (generated by the fixturing sequences and/or the machining operations) that may be introduced by a non-effective clampinglocator system (especially when hyper static constraints conditions and/or non closure-force conditions and/or non perfect clamping equilibrium arise).

\section{THE ADAPTIVE FIXTURING: WORKING PRINCIPLE}

Here an innovative adaptive fixture (see Fig. 1) based on active clamping devices (which integrates smart actuator) with adaptronic features has been designed and developed.

The principle consists of an automatic recovering of work-

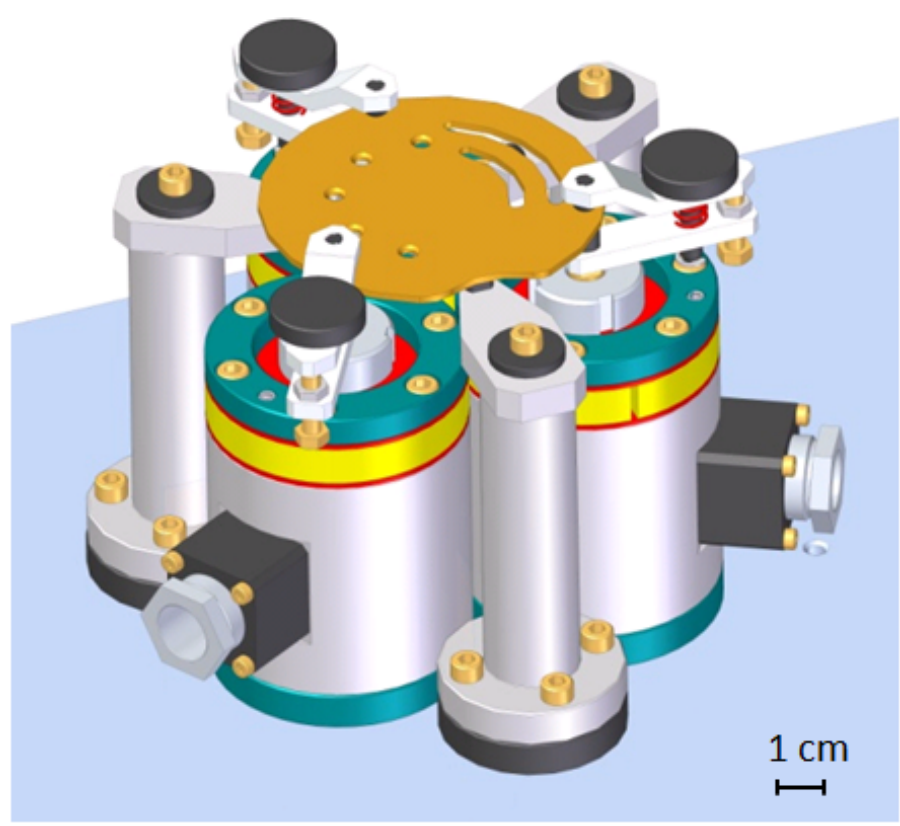

FIGURE 1. FIXTURING SYSTEM DESIGN.

piece positioning errors and distortions based on active/dynamic clamping forces. The control loop is closed through smart sensors integrated in the clamping device itself. Trade-off studies (max. strokes vs. size vs. stiffness vs. bandwidth) have been done to select the proper actuators and guiding (frictionless) system.

The adaptive fixturing is based on the Active Clamps concept. The approach consists in dividing the clamping sequence in three steps as follows(see Fig. 2):

1. Pliers opportunely guided but with no axial constraint.

2. Clamping of pliers, which can adapt themselves to the workpiece without inducing unwanted constrains to it.

3. Blocking of the axial degree of freedom of pliers, in order to constrain the workpiece with respect to the fixture.

In the second step, the degree of freedom left free is along the clamping direction. Indeed, if the vertical degree of freedom of pliers during clamping phase is free, the clamping does not load the workpiece vertically. If pliers are guided in their vertical movement, reducing as much as possible friction along guides, they can adapt their vertical position, remaining "hung up" to the workpiece. In the third step, the position of the pliers is opportunely constrained to the base.

This procedure should leave the workpiece without any residual stress after being clamped. Supposing that the procedure is not correctly executed and the workpiece is deformed, an actuator embedded inside the active clamp module (see Fig. 2, 


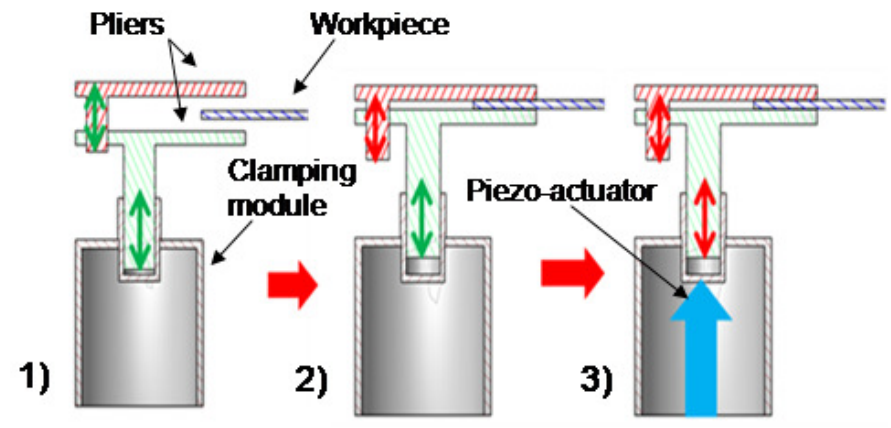

FIGURE 2. CLAMPING PRINCIPLE.

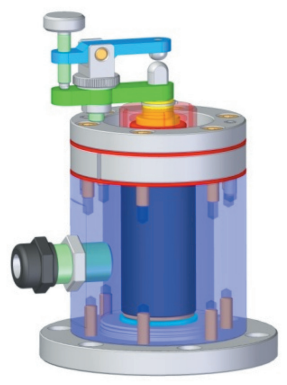

FIGURE 3. 3D CAD MODEL OF THE ACTIVE CLAMPING MODULE.

3), and Fig. 3) may restore the undeformed configuration. If the cause of the anomaly is the clamping procedure, the force which deforms the workpiece will be generated by the clamping module itself. In this situation, a counter force, provided by an actuator, may cancel the effects of the distortion.

The ideal procedure explained above is applied by the components of the clamping module. The clamps which are constrained to the adjustable moveable rod are free to move one with respect to the other and they both follow the vertical movement of the rod. The rod is free to move vertically with respect to the rest of the module. In order to clamp a workpiece the relative movement of the clamps is first constrained to the workpiece so that the clamps, the moveable rod and the workpiece constitute a single body. Until the rod is not constrained to the rest of the clamping module, it is free to move vertically and the workpiece does not experience any deformation. Then, the rod is fixed to the flexures. The user should simply turn the ring nut which deform the blocking chuck until it completely constrains the moveable rod. Now the workpiece is fully constrained. The piezoactuator is able to exert his action. The fixturing system is now an active system. Resuming, the module has some main components which are:
- Clamps: needed to fix the workpiece, they have a quite relative freedom to move one with respect to the other in the vertical direction;

- Adjustable moveable rod: the clamps are fixed to the rod, while the rod is free to move vertically with respect to the rest of the module;

- Flexures: mechanical filters for shear forces and momentum to prevent the piezoactuator from cracking;

- Blocking chuck and ring nut: mechanical devices needed to clamp the rod to the flexures;

- Axial Spring: the spring push up the rod until it reaches its maximum allowed stroke. The stiffness is really low in order not to oppose resistance when the workpiece is positioned.

\section{THE ADAPTIVE FIXTURING: DESIGN}

For designing the new fixturing system several crucial aspects have been analysed and solved, as reported in the following.

\section{The actuation}

The choice of the actuator was the most relevant and crucial task of the whole design as it had relevant influence on the further mechanical design as will be described later. Many actuators are claimed to be smart and they offer different features (see Tab. 1). The most important ones for this application are the efficiency, the bandwidth, the power density and the "passive" mechanical stiffness and compactness.

Another critical key issue for the actuator choice and for the rest of the mechanical design is the knowledge of the magnitude of the machining forces. At the end of the trade off analysis, the best suited solution was the piezoelectric actuator.

\section{The stroke}

Modularity and flexibility are main requirements for an adaptive fixture. Indeed, a really valid clamping module must be able to clamp different workpieces in different clamping points (see Fig. 2, 2)) The active clamping module has to provide the stroke and force required to recover the distortion errors eventually introduced on the workpiece during clamping steps.

Since the workpiece may present clamping points at different heights, the new "active" clamping device still is required to reach, engage (and disengage) clamping points of different workpieces. Due to the fact that piezoactuators provide limited stroke, a special hybrid mechanism has been designed and introduced in the clamp to fulfill also the later requirement. This mechanism lets the moveable rod move with respect to the rest of the fixed part of the clamping module until the user decides to constrain the workpiece as described before.

The active clamping module can be equipped also with a non-contact "eddy current" sensor to measure the position of the clamping point (this will enable the automatic recovering in closed loop of error generated by clamping procedures itself). 
TABLE 1. SMART ACTUATORS COMPARISON.

\begin{tabular}{|c|c|c|c|c|}
\hline $\begin{array}{l}\text { ACTUATION } \\
\text { METHOD }\end{array}$ & EFFICIENCY & SPEED & $\begin{array}{l}\text { POWER } \\
\text { DENSITY }\end{array}$ & STIFFNESS \\
\hline $\begin{array}{l}\text { ELECTRO- } \\
\text { MAGNETIC }\end{array}$ & High & Fast & Medium & $\begin{array}{l}\text { High (in ac- } \\
\text { tive loop) }\end{array}$ \\
\hline $\begin{array}{l}\text { PHASE } \\
\text { CHANGE }\end{array}$ & Very High & Medium & Medium & Low \\
\hline $\begin{array}{l}\text { PIEZO- } \\
\text { ELECTRIC }\end{array}$ & High & $\begin{array}{l}\text { Very } \\
\text { Fast }\end{array}$ & High & Very High \\
\hline $\begin{array}{l}\text { SHAPE } \\
\text { MEMORY }\end{array}$ & Low & Medium & Very High & Medium \\
\hline $\begin{array}{l}\text { MAGNETO- } \\
\text { STRICTIVE }\end{array}$ & Medium & Medium & High & High \\
\hline $\begin{array}{l}\text { ER-EF FLU- } \\
\text { IDS }\end{array}$ & Medium & $\begin{array}{l}\text { Medium } \\
\text { /Low }\end{array}$ & $\begin{array}{l}\text { Medium } \\
\text { /Low }\end{array}$ & Low \\
\hline
\end{tabular}

The piezoactuator integrates also a strain gauge sensor and a force sensor.

\section{The mechanical filter}

A piezoelectric actuator cannot react shear forces and momentum, in fact, under these kind of stresses, it would simply crack. Consequently, the piezoactuator must be interfaced to the rest of the world by means of a mechanical system able to decouple the loads applied. For this purpose it has been developed a self-guided piezo-based actuator (patent pending, Applicant Ce.S.I.), which can support radial loads, torque/momentum, while providing high axial and lateral stiffness.

\section{Compactness of the solution}

One of the disadvantages that affect the existing adaptive fixturing devices is the size. They are really huge system, difficult to be integrated in different grinding machines. On the other hand, big dimensions imply, most of the times, a low bandwidth. Smaller clamping modules can find more applications in different fixtures.

\section{SIMULATION AND EXPERIMENTAL VALIDATION Mechatronic numerical model}

The fixturing system may be schematized as showed in Fig. 4 and Fig. 5. The fixturing system is constrained in the XY plane movement and in the rotation around $\mathrm{Z}$ axis. Due to the vertical movement of the actuators it can rotate around $\mathrm{X}$ and $\mathrm{Y}$ axis and move along $\mathrm{Z}$ axis.

As the fixturing system has three supported points, it can be modeled by the canonic mechanical equations' system. Supposing the inertial frame reference of the fixturing system is the one showed in Fig. 4 (thus the workpiece mass should be negligible with respect to the fixturing system mass), the equation of motion are the following

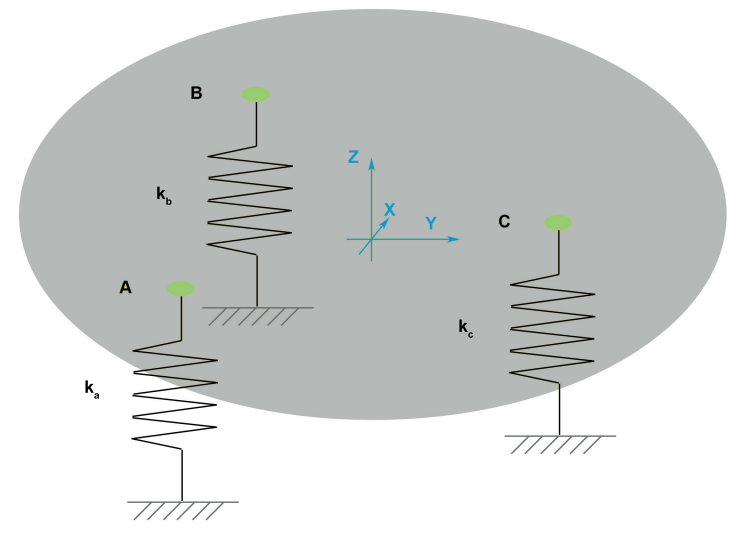

FIGURE 4. MODEL OF THE FIXTURING SYSTEM: THREE SPRINGS CONNECTED TO A RIGID BODY.

$$
\begin{aligned}
m \ddot{z} & =\sum F_{z} \\
I_{x} \dot{\omega}_{x} & =\sum M_{z} . \\
I_{y} \dot{\omega}_{y} & =\sum M_{y}
\end{aligned}
$$

Where $m$ is the fixturing system mass, $I_{x}$ and $I_{y}$ and $M_{x}$ and $M_{y}$ are respectively the main inertias and the moments with respect to (w.r.t.) the $X$ and $Y$ axes, $F_{z}$ is the sum of all the vertical forces loading the fixturing system. Supposing the angles little enough, it can be assumed that

$$
\begin{aligned}
& \omega_{x}=\dot{\alpha}_{x} \\
& \omega_{y}=\dot{\alpha}_{y}
\end{aligned}
$$

where $\alpha_{x}$ and $\alpha_{y}$ are the Euler's angles of the inertial frame w.r.t. the main frame.

The dynamic equation (1) may be resumed in matrix form

$$
\mathbf{M} \ddot{\mathbf{z}}=\mathbf{K z}+\mathbf{E F}
$$

where the vector $z$ entries are the degrees of freedom of the fixturing system

$$
\mathbf{z}=\left[\begin{array}{c}
z_{c} \\
\alpha_{x} \\
\alpha_{y}
\end{array}\right]
$$




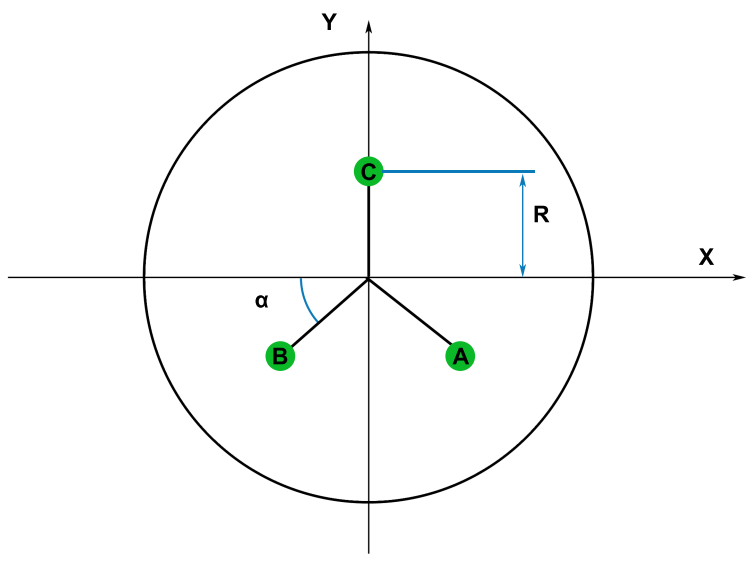

FIGURE 5. FIXTURING SYSTEM, LABELS FOR THE CLAMPING MODULES.

The mass matrix is a diagonal matrix and assumes the following form

$$
\mathbf{M}=\left[\begin{array}{ccc}
m & 0 & 0 \\
0 & I_{x} & 0 \\
0 & 0 & I_{y}
\end{array}\right]
$$

while the stiffness is, according to Fig. 4 and 5

$$
\mathbf{K}=\left[\begin{array}{ccc}
-k_{a+b+c} & R s \alpha k_{a+b}-k_{c} R & R c \alpha k_{a-b} \\
R s \alpha k_{a+b}-k_{c} R & -R^{2}\left(s^{2} \alpha k_{a+b}-k_{c}\right) & R^{2} c \alpha s \alpha k_{a-b} \\
-R c \alpha k_{a-b} & R^{2} c \alpha s \alpha k_{a-b} & -(R c \alpha)^{2} k_{a+b}
\end{array}\right]
$$

where $k_{a+b+c}=k_{a}+k_{b}+k_{c}, k_{a+b}=k_{a}+k_{b}, k_{a-b}=k_{a}-k_{b}$ $s \alpha=\sin (\alpha), c \alpha=\cos (\alpha)$.

The matrix $\mathbf{E}$ relates the vertical forces exerted by the piezoactuators to the degrees of freedom of $z$ (4)

$$
\mathbf{E}=\left[\begin{array}{ccc}
1 & 1 & 1 \\
-R s \alpha & -R s \alpha & R \\
R c \alpha & -R c \alpha & 0
\end{array}\right]
$$

and, finally, the force vector

$$
\mathbf{F}=\left[\begin{array}{c}
F_{A} \\
F_{B} \\
F_{C}
\end{array}\right]
$$

It has been realised a finite element(FE) model of the clamping module, so that $k_{a}, k_{b}$ and $k_{c}$, the vertical stiffness of the modules, account also for the piezoactuator stiffness and $\mathbf{F}_{\mathbf{A}}, \mathbf{F}_{\mathbf{B}}, \mathbf{F}_{\mathbf{C}}$, the force exerted by the modules, respectively labelled A, B, C in Fig. 5.

The above system could be easily implemented in the linear state space representation

$$
\begin{aligned}
& \ddot{\mathbf{x}}=\mathbf{A x}+\mathbf{B u} \\
& \mathbf{y}=\mathbf{C x}+\mathbf{D u}
\end{aligned}
$$

where $\mathbf{x}=\left[\begin{array}{llllll}\dot{\mathbf{z}}_{\mathbf{c}} & \dot{\alpha}_{\mathbf{x}} & \dot{\alpha}_{\mathbf{y}} & \mathbf{z}_{\mathbf{c}} & \alpha_{\mathbf{x}} & \alpha_{\mathbf{y}}\end{array}\right]^{\mathbf{T}}, \mathbf{u}=\left[\begin{array}{lll}\mathbf{F}_{\mathbf{a}} & \mathbf{F}_{\mathbf{b}} & \mathbf{F}_{\mathbf{c}}\end{array}\right]^{\mathbf{T}}$ and $\mathbf{A}=$ $\left[\begin{array}{cc}-\mathbf{M}^{-\mathbf{1}} \mathbf{D}_{\mathbf{a}}-\mathbf{M}^{-\mathbf{1}} \mathbf{K} \\ \mathbf{I} & \mathbf{0}\end{array}\right], \mathbf{B}=\left[\begin{array}{c}-\mathbf{M}^{-\mathbf{1}} \\ \mathbf{0}\end{array}\right], \mathbf{C}=\mathbf{I}, \mathbf{D}=\mathbf{0}$, with damping matrix $\mathbf{D}_{\mathbf{a}}$ composed according to the Rayleigh modal damping, $\mathbf{D}_{\mathbf{a}}=\gamma \mathbf{M}+\beta \mathbf{K}, \gamma$ and $\beta$ assume values in order to present critical modal damping ratios between 0.01 and 0.05 .

The state space model is a linear representation of the fixturing system which can be embedded inside Simulinks environment. It is possible to study several control schemes and the dynamic of the overall system (fixturing system, amplifier, actuator, sensors, ...).

In the Fig. 6 is showed the Simulink's scheme of the numerical model representing the complete system. It has been chosen a three SISO (single input single output) decentralized co-located control scheme, where every loop is a single PID (Proportional-Integral-Derivative) controller. The scheme shows how the model has been developed, every single loop has its own sensor's and amplifier's dynamic and its own PID controller.

The numerical model is represented by Fig. 7. The sensors are placed as close as possible to the actuation points and the correspondent conditioned electrical voltage signal is sent to the controller block that drives the actuator according to the designed control strategy.

The controller chosen is a PI (Proportional-Integral), as this first application is meant to be quasi-static so that the derivative contribution is not needed. In the case the fixturing should be used for active vibration control (AVC), then not only the derivative action would be needed but should be the major contribution to the control in order to ensure stability [6]. The goal of the simulation is the recovery of the undeformed configuration of the workpiece by means of the action of the three clamping modules in a relatively short time.

When the modelled static disturbance force $(F)$ is applied over a clamped point $(C 1)$, the workpiece is deformed and the $C 1$ point moves. This displacement is meant to simulate the effect of an incorrect clamping procedure or the action of an external disturbance. After few moments, the closed loop control is activated. Easily the PI controller compensates the displacement and very quickly the workpiece is completely restored in its undeformed configuration. 


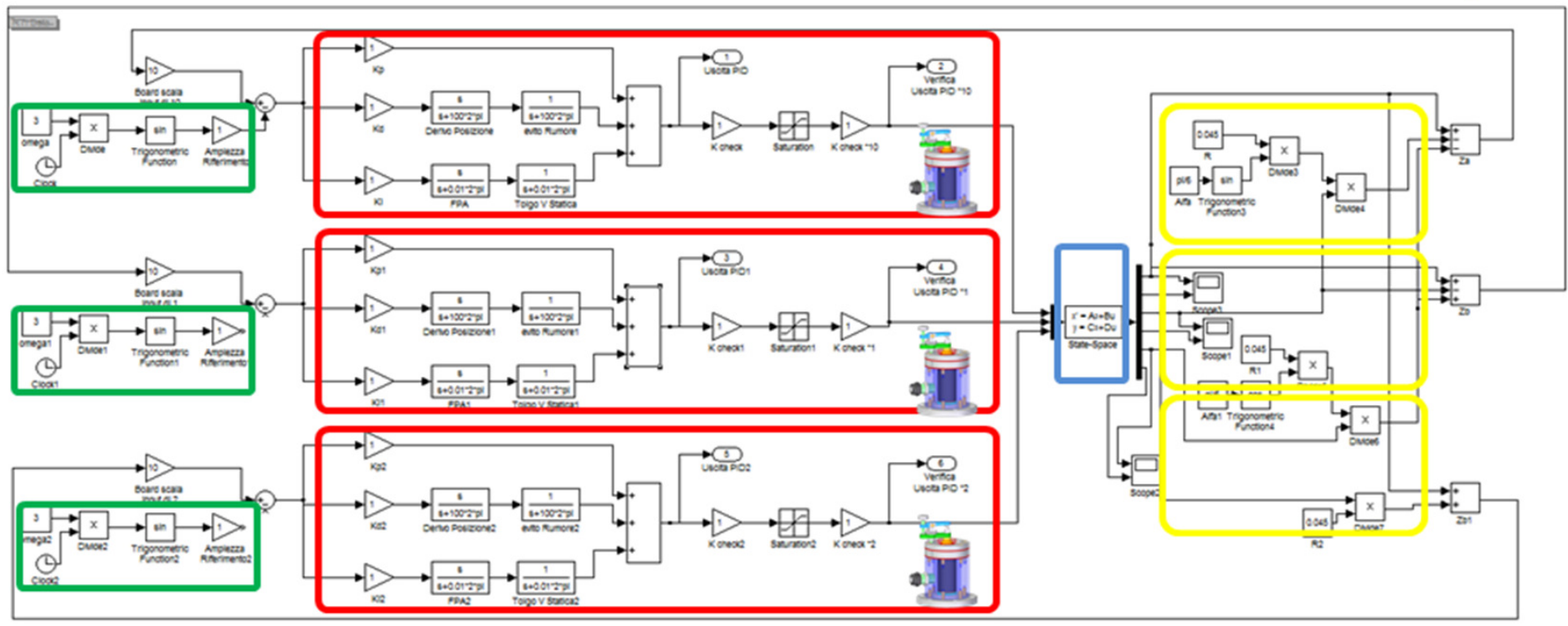

FIGURE 6. SIMULINK'S MODEL OF THE FIXTURING MODEL.

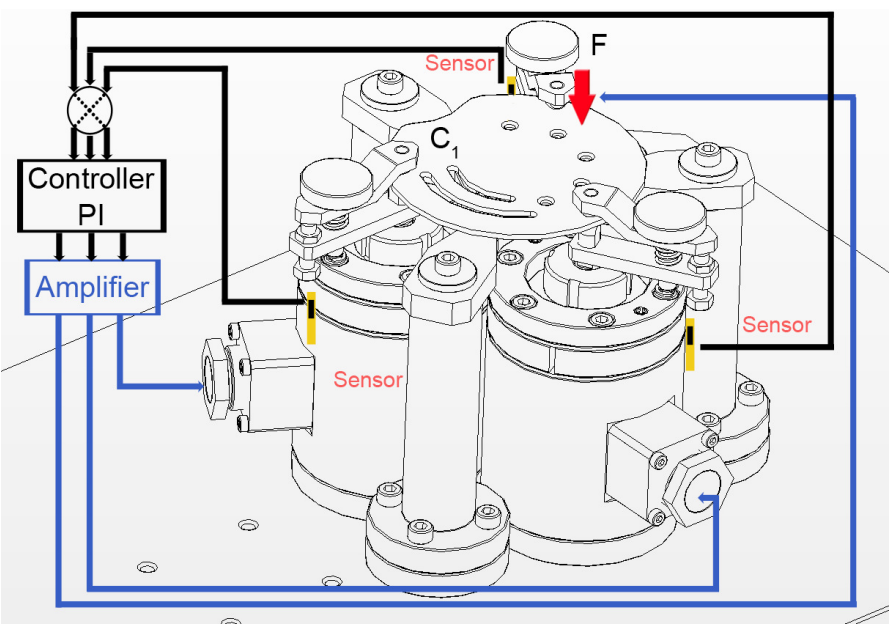

FIGURE 7. SETUP FOR MECHATRONIC SIMULATION.

The mechatronic simulation of the system has been performed in Matlab $R$ / Simulink $R$ environment: the result is shown in Fig. 9 (dashed line).

\section{Experimental tests}

Once the numerical simulation confirmed the working principle, experimental tests on the physical test bench have been carried out (see Fig. 8). The test bench was equipped with an electronic board (controller) programmed through the algorithms

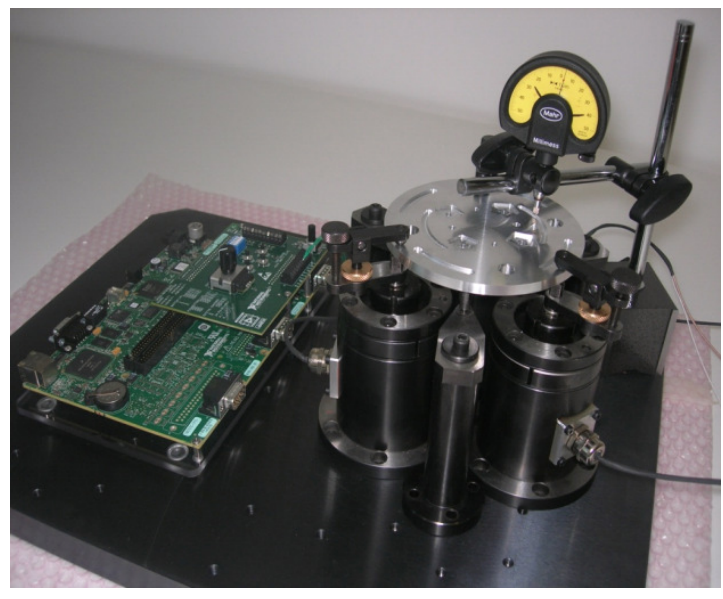

FIGURE 8. EXPERIMENTAL SETUP.

defined and tuned within the previous mechatronic simulations. The workpiece is initially positioned upon the three datum points in order that it results aligned in vertical direction and all sensors measures can be consequently reset to zero. The workpiece is then clamped and a positioning error is imposed through the application of a vertical asymmetric force on it: the resultant workpiece misalignment is detected by the sensors. When the PI control is switched-on this error is immediately recovered by the active clamps and the initial alignment restored. The experimental recovering curve of one clamp is shown in Fig. 9 (continuous 


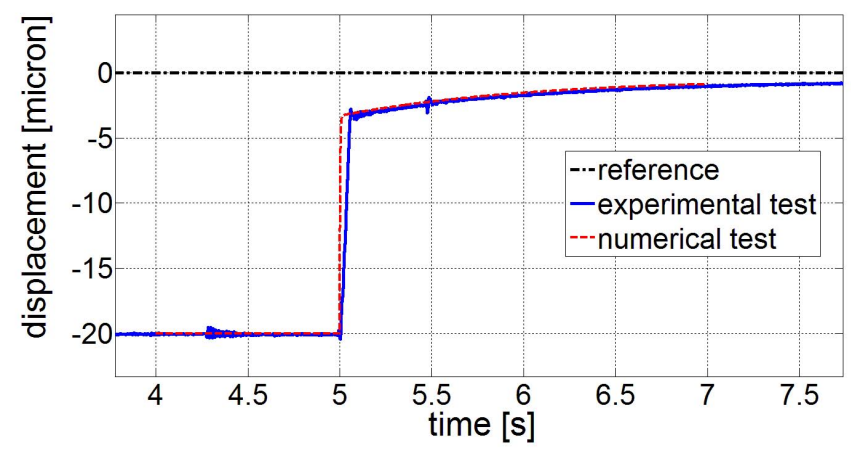

FIGURE 9. NUMERICAL VS. EXPERIMENTAL RESULTS: CONTROL SWITCH ON AT $\mathrm{t}=5 \mathrm{~s}$.

line). In the figure the control has been switched on after 5 seconds. The first ramp is due to the proportional effect of the PID, while the following slow error reduction is due to the integral contribution. It can been seen that test results have validated the numerical simulation as well as definitely proven the effectiveness of the proposed system.

\section{CONCLUSIONS}

An adaptive fixturing system, for ultra high precision micropositioning of thin walled precision parts based on an active clamping module has been developed and prototyped. The active clamps concept has been presented and explained.

The proposed adaptive fixturing is able to overcome the drawbacks of the conventional fixture adding new capabilities, like automatic recovering of clamping errors and workpiece distortions. The design concept enables the possibility to easily reconfigure the fixtures for different workpieces size, preserving high performances in terms of precision, stiffness and compactness.

The working principle has been verified by numerical mechatronic simulations end experimental tests as well. These results are very promising as they prove not only the engineering concept, but also the proper fabrication (in accordance to expectations, in particular regarding stiffness and ultra precise micromovements) of the active clamps. In addition the device can be potentially used for vibration chatter suppression during machining operation.

This novel adaptive fixturing system will represent a challenging improvement in micromachining of thin walled parts and could open interesting perspectives for the industrial applications of microadaptronics in manufacturing sector.

\section{ACKNOWLEDGMENT}

The work carried out in this report is partially funded by the EU Project IP 214013 titled Integ-Micro. The authors wish to thank the European Commission and all the partners of the consortium.

\section{REFERENCES}

[1] Denkena, B., Immel, J., and Götz, T., 2005. "Drehspannfutter mit integrierter, mechatronischer feinpositioniervorrichtung”. In Internationales Forum Mechatronik, Augsburg, Deutschland.

[2] Haase, R., Seewig, J., and Reithmeier, E., 2005. "Verbesserte hartfeinbearbeitung von zahn rädern”. Bearbeitungsaufma $\beta$ präzisionsge schmiedeter Zahnräder für den Schleifprozess optimieren, wt Werkstattstechnik online, 95(6), pp. 447-452.

[3] Eckart, U., Pingfa, F., and Stefan, D., 2004. "Dynamic clamping force for jaw-chucks for high speed cutting". Production Engineering, 11(1), pp. 149-152.

[4] MAFFIX Project COP-CT-2006-032818, F., 2006. Completely flexible and reconfigurable fixturing of complex shaped workpieces with mrf. Tech. rep., EU commission.

[5] Nee, A. Y. C., 2003. "Computer-aided tooling design for manufacturing processes". Innovation in Manufacturing Systems and Technology (IMST).

[6] Preumont, A., 2002. Vibration control of active structures: an introduction, Vol. 96. Springer. 